\title{
Modeling airfield ground lighting systems for narrow-band power-line communications
}

\author{
Joaquín Granado, Jorge Chávez, Antonio Torralba and Ana Cinta Oria
}

\begin{abstract}
Airfield Ground Lighting (AGL) systems are responsible for providing visual reference to aircrafts in the airport neighborhood. In an AGL system, a large number of lamps is organized in serial circuits, and connected to current regulators that supply energy to the lamps.

Controlling and monitoring lamps (including detection and location of burnt-out lamps) are critical for cost-saving maintenance and operation of AGL systems. Power-line Communications (PLC) is an attractive technology to connect elements of the AGL, reusing the power distribution cable as a transmission medium. PLC technologies avoid the installation of new wires throughout the airport infrastructure.

This paper proposes a new model for power-line communication links in AGL systems. Every element (isolation transformer, primary circuit cable, and lamps) has been analyzed in laboratory and modeled using SPICE. The resulting models have been integrated to build a complete power-line link model. Simulation results are compared to experimental results obtained in real conditions in the Airport of Seville (Spain).
\end{abstract}

Index Terms-- Aircraft landing guidance, lighting control, power distribution, power-line communication, airports, airfield lighting systems.

\section{INTRODUCTION}

$\mathrm{W}$ orldwide trends in aviation industry indicate that monitoring and controlling airfield lighting systems increase the airport capacity and operation efficiency, improving the safety in both the air traffic controller and the aircrafts [1], [2].

Airport Airfield Ground Lighting (AGL) systems are in charge of emphasizing the runway, giving visual reference of speed and alignment to aircrafts in the final stages of approaching, landing and taxiing operations [3].

The so-called marker lights, or beacons, are lamps installed in devices, which modify the pattern, intensity, color and direction of the light emission. The number of beacons is variable (from 500 in small airports, up to 3000-4000 in the larger ones) and involves an enormous and expensive work of maintenance and conservation, mainly derived from the detection and location of burnt-out lamps. These tasks must be done in-situ, since most of the beacons are directional (or

This work was partially supported by the Spanish Ministry of Science and Technology under project TEC2008-06881-C03-02/TEC, ELIMCO and the Andalousian regional government.

Authors are with Dpto. de Ingeniería Electrónica. Universidad de Seville. Escuela Superior de Ingenieros. Av. Descubrimientos s/n. 41092-Seville. Emails: \{joaquin, chavez, torralba, cintalep\}@ zipi.us.es. bidirectional). Additionally, the International Civil Aviation Organization (ICAO) [3] and the Federal Aviation Agency (FAA) [4] impose security restrictions (maximum number of burnt-out lamps, etc.). As a result, the runway has to be out of service during significant periods of time due to maintenance operations in the AGL systems. Therefore, this scenario has stimulated the development of new technologies to facilitate these tasks.

The hard operation conditions of the runway, as well as the reluctance of the airport authority to install new cables, avoid the development of wired-based solutions. Wireless technologies are also rejected due to possible interference in radio navigation instruments. In this situation, the power-line communications (PLC) systems over AGL become an attractive choice. PLC technologies reuse the same power distribution cable to provide data communication, mainly control and supervision, avoiding the installation of new cables.

There are some solutions in the marketplace that use PLC technology [5] in AGL, and some experimental results have been published [6]-[7]. However, there is still a lack of proper models to allow planning and deployment of these systems, especially when compared to conventional PLC applications such as in-door or access technologies.

The objective of this paper is twofold: to propose a complete simulation model for narrow-band PLC links in AGL power-lines, as well as to evaluate the impact of the AGL elements in the communications system. The resulting simulation model will help engineers to property design, maintain and operate AGL systems based on PLC technologies. Experiments performed in the airport of Seville (Spain) validate these models.

This paper is organized as follows. Section II describes the AGL system, as well as the topology proposed for PLC links. In section III the main elements of the AGL system are analyzed in order to derive a SPICE model. In section IV these models are combined to build a complete PLC link. Simulations results are also compared to experimental measurements in section IV. Section V discusses some design considerations. Finally some conclusions are drawn in Section VI.

\section{SYSTEM ARCHITECTURE}

\section{A. System description}

Fig. 1 depicts the basic structure of the AGL system. Detailed specifications of the visual aids and the airport electrical systems can be found in [3] and [8], respectively. 


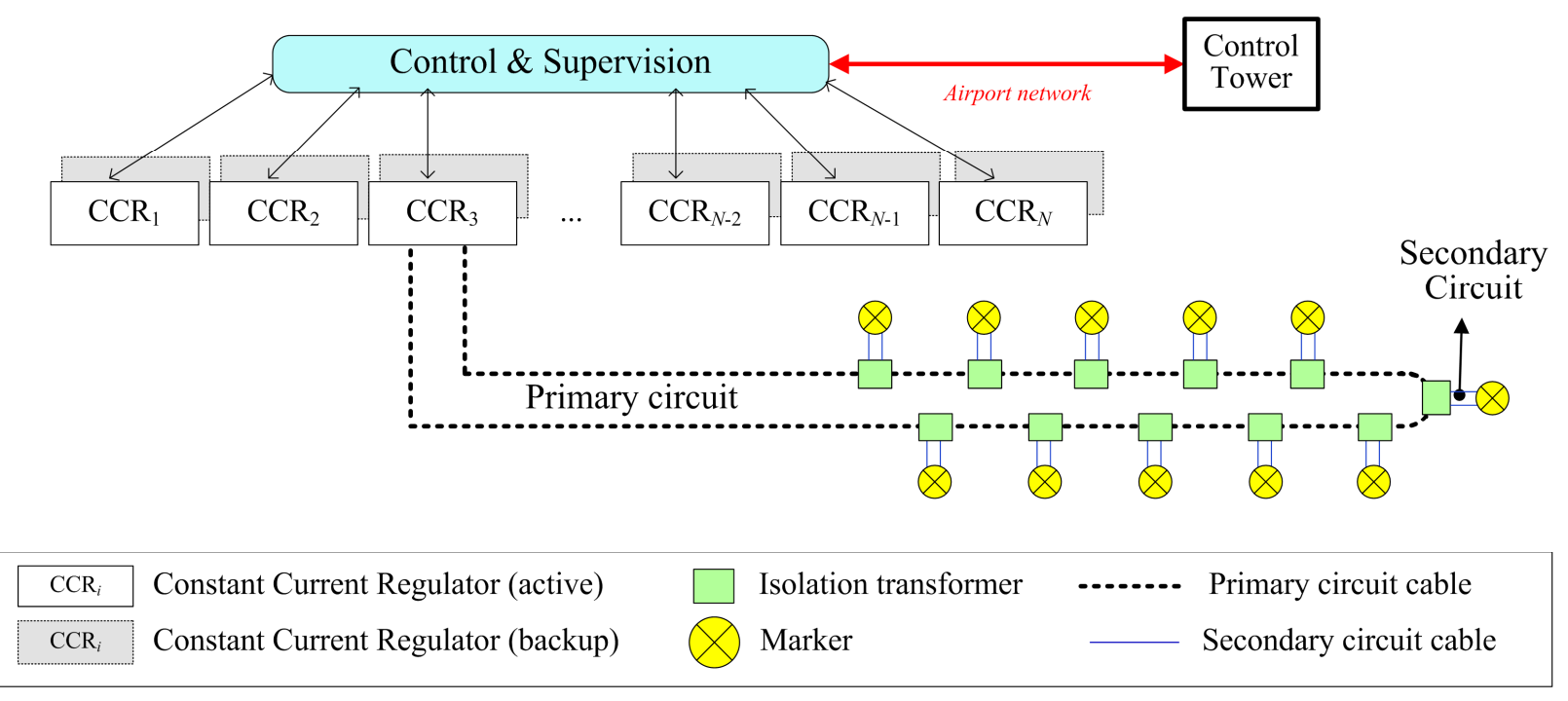

Fig. 1. Airfield ground lighting system (AGL). Constant current regulators (CCR) are located in the regulators room. Primary circuit spreads throughout the airfield ground. Every secondary circuit connects one lamp to the isolation transformer (IT).

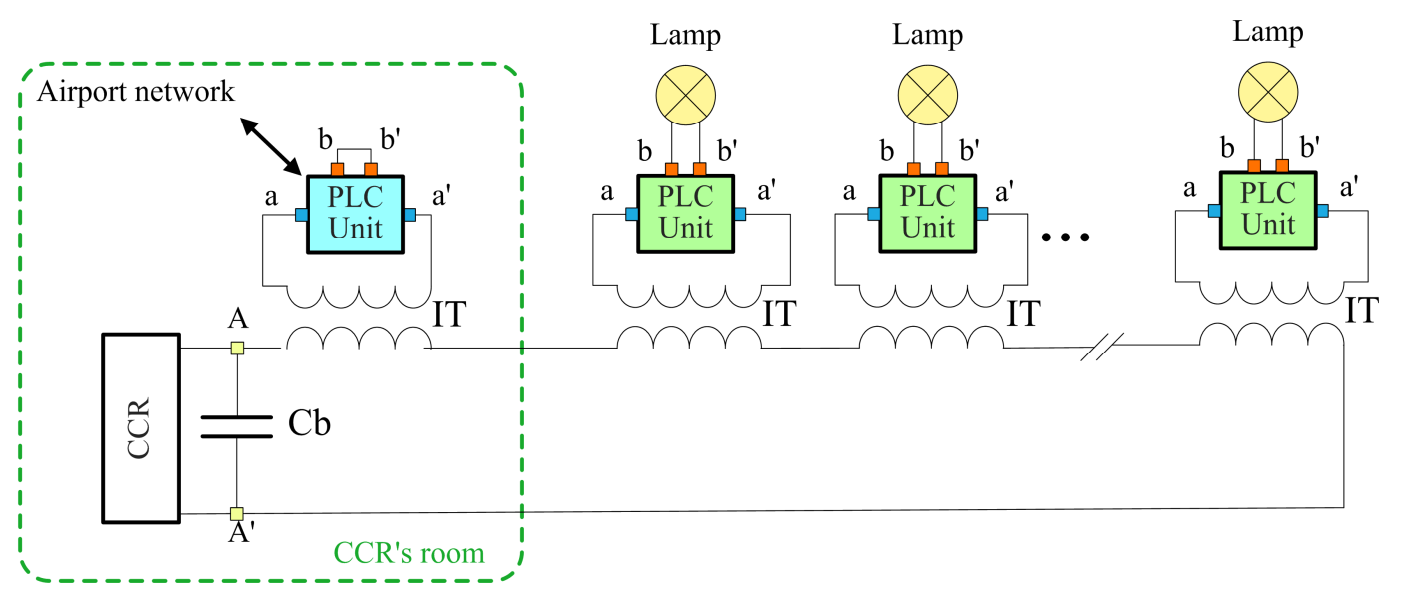

Fig. 2. Selected topology: the PLC unit is connected to the primary power circuit using an isolation transformer (IT) through the port aa', and the lamp is monitored and controlled by means of port bb'.

Constant Current Regulators (CCR's) are placed in the regulators room, which is usually located in the neighborhood of the airport electrical power supply. The CCR is in charge of supplying a constant-tunable sinusoidal current to the primary power circuit. The beacon brightness can be adjusted, either manually or remotely, by means of controlling the RMS value of the electrical current provided by the CCR.

The CCR is series connected to a single-core cable, the primary circuit cable, which distributes the generated current to the lamps through the isolation transformer (IT). The IT maintains the continuity of the series circuit, even when one lamp is burnt-out. The IT also provides isolation from the high voltages in the primary circuit.

The secondary power circuits use bipolar cables to connect the lamps to the ITs.

There are many types of beacons that can be grouped according to their functionality in the following categories [3]: Approach Lighting System (ALS) \& Precision Approach Path Indicator (PAPI) which provide pilots with the necessary information to perform visual approaching; Runway Lighting System (RLS), which consists of the runway centerline, edge, threshold, and end lights; and taxiway and apron lights, and guidance signs, which help the pilot in the taxiing maneuver.

\section{B. Topology}

The PLC technology on the AGL power distribution system allows communication between connected components reusing the already existing cables.

We have selected a star topology, where the lamps are homed into a central hub that manages the access to the media. The central hub is located in the CCR room, and connected to the airport network. The communication is assumed to be semi-duplex, and no repeater is considered in our analysis.

Due to the reduced bandwidth required for control and monitoring, there are some commercial narrow-band PLC modems that fit the system requirements. These modems typically operate in frequencies from tens to a few hundreds of $\mathrm{kHz}$. 
The PLC modems are installed in the secondary circuits (see Fig. 2), using a parallel branch, rather than in the primary circuit. This topology is repeated in the power header, in the regulator room, where a PLC modem is connected by means of an isolation transformer to the primary circuit. The selected topology is safe, if one element of the PLC network fails, it would be isolated of the primary circuit and, consequently, would not affect the normal operation of the AGL components.

The selected topology is safer, cost-effective and ensures minimum interference with the normal AGL operation. An alternative serial topology, i.e. connecting the modems to the primary circuit using inductive couplers, would require to manipulate the cable shield. In addition, the inductive couplers are expensive components (every beacon requires one coupler, and up to several hundred beacons can be found in a circuit). However, this alternative could be used to connect the modem located in the regulator room, since the primary cable is available in the CCR rear connector.

Fig. 3 describes the topology of the PLC unit. A common mode choke $\mathrm{Lb}$ is implemented to reduce the impact of the high frequencies in the lamp. This component behaves as a low pass filter, presenting high impedance at PLC carrier frequency, and reducing those power components to be dissipated in the lamp. Conversely, the capacitor Cs attenuates the high amplitude of the power signal, located at $50 \mathrm{~Hz}$, at the modem input. The signal transformer ST is in charge of coupling the PLC modulated signal to the secondary circuit.

The topology described in Fig. 3 also includes a switched power supply to provide energy to PLC unit, and a relay to switch on or off the lamp.

The PLC modem unit has been prototyped using a development board from Renesas (YSDK-PLC-EU-B) that includes a Yitran IT800 PLC transceiver.

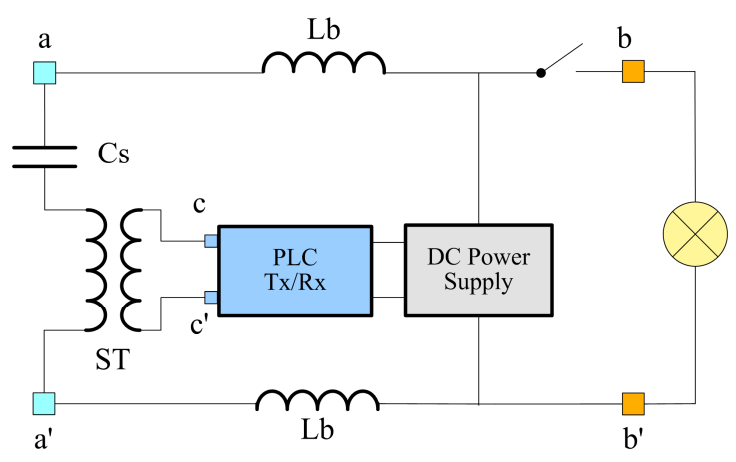

Fig. 3. PLC modem unit.

\section{PROPOSED MODELS}

\section{A. Isolation transformers (ITs)}

\section{1) Experiments description}

We have used two different isolation transformers in our experiments: an old transformer manufactured by ADB-Air and a type ring-core series transformer (RST) manufactured by ADB-Siemens. Both components are ICAO and FAA compliant ([8], [9]).
The first IT, termed type-1, is no longer commercially available, but it has been installed in many airports since the sixties. The second IT, termed type-2, is widely used in new airports. Both isolation transformers have been analyzed using a Vector Network Analyzer (VNA).

We have measured three parameters in the $(80-200) \mathrm{kHz}$ frequency range: the short-circuit impedance Zsc (the VNA is connected to the primary port and the secondary port is shortcircuited); the open-circuit admittance Yoc (the VNA is connected to the primary port and the secondary port is opencircuited); and the insertion losses (or $S_{21}$ scattering parameter). Tables I and II show the measured impedances and admittances for both ITs at 80, 120, 160 and $200 \mathrm{kHz}$. Fig. $4 \mathrm{a}$ and $4 \mathrm{~b}$ depict the measured insertion losses.

TABLE I

MEASURED SHORT-CIRCUIT IMPEDANCE FOR TYPE-1 AND TYPE-2 ISOLATION TRANSFORMERS

\begin{tabular}{|c|c|c|c|c|}
\cline { 2 - 5 } \multicolumn{1}{c|}{} & \multicolumn{2}{c}{ type-1 transformer } & \multicolumn{2}{c|}{ type-2 transformer } \\
\hline $\begin{array}{c}\boldsymbol{f} \\
{[\mathbf{k H z}]}\end{array}$ & $\begin{array}{c}\mathbf{r e a l}(\mathbf{Z s c}) \\
{[\boldsymbol{\Omega}]}\end{array}$ & $\begin{array}{c}\text { imag(Zsc) } \\
{[\boldsymbol{\Omega}]}\end{array}$ & $\begin{array}{c}\text { real(Zsc) } \\
{[\mathbf{\Omega}]}\end{array}$ & $\begin{array}{c}\text { imag(Zsc) } \\
{[\boldsymbol{\Omega}]}\end{array}$ \\
\hline \hline 80 & 62 & 408 & 25 & 94 \\
\hline 120 & 102 & 629 & 34 & 135 \\
\hline 160 & 156 & 897 & 43 & 176 \\
\hline 200 & 237 & 1255 & 53 & 219 \\
\hline
\end{tabular}

TABLE II

MEASURED OPEN-CIRCUIT ADMITTANCE FOR TYPE-1 AND TYPE-2 ISOLATION TRANSFORMERS

\begin{tabular}{|c|c|c|c|c|}
\cline { 2 - 5 } \multicolumn{1}{c|}{} & \multicolumn{2}{c|}{ type-1 transformer } & \multicolumn{2}{c|}{ type-2 transformer } \\
\hline $\begin{array}{c}\boldsymbol{f} \\
{[\mathrm{kHz}]}\end{array}$ & $\begin{array}{c}\text { real(Yoc) } \\
{\left[\mathbf{\Omega}^{-1}\right]}\end{array}$ & $\begin{array}{c}\text { imag(Yoc }) \\
{\left[\mathbf{\Omega}^{-1}\right]}\end{array}$ & $\begin{array}{c}\text { real(Yoc) } \\
{\left[\mathbf{\Omega}^{-1}\right]}\end{array}$ & $\begin{array}{c}\text { imag(Yoc }) \\
{\left[\mathbf{\Omega}^{-1}\right]}\end{array}$ \\
\hline \hline 80 & $4.4 \mathrm{e}-4$ & $-2.8 \mathrm{e}-4$ & $8.5 \mathrm{e}-4$ & $-8.3 \mathrm{e}-4$ \\
\hline 120 & $3.5 \mathrm{e}-4$ & $-1.3 \mathrm{e}-4$ & $6.7 \mathrm{e}-4$ & $-5.4 \mathrm{e}-4$ \\
\hline 160 & $3.0 \mathrm{e}-4$ & $-1.3 \mathrm{e}-5$ & $5.6 \mathrm{e}-4$ & $-3.4 \mathrm{e}-4$ \\
\hline 200 & $2.6 \mathrm{e}-4$ & $9.2 \mathrm{e}-5$ & $4.9 \mathrm{e}-4$ & $-1.6 \mathrm{e}-4$ \\
\hline
\end{tabular}

\section{2) Results}

The short-circuit complex impedance of the type-1 transformer (see Table I) exhibits both a resistive and an inductive behavior. The resistance is in the interval [62-237] $\Omega$ whereas the measured serial inductance varies in the range [0.81-1.00] $\mathrm{mH}$ in the frequency band of interest.

The short-circuit complex impedance of the type-2 transformer also exhibits a resistive and an inductive behavior (see Table II). The resistance is in the interval [25-52] $\Omega$, which is smaller than the resistance of the type- 1 transformer, whereas the measured serial inductance varies in the range [0.19-0.17] $\mathrm{mH}$ in the same frequency band.

Note that the resistive behavior of both transformers is not due to the skin effect, which shows a $f^{0.5}$ dependence.

The measured open-circuit admittance is presented in Table II. The real part of the admittance shows a resistive behavior due to skin effect (increasing with $f^{0.5}$ ). The measured conductance for the type- 1 transformer is smaller than the results obtained for type- 2 transformer. 


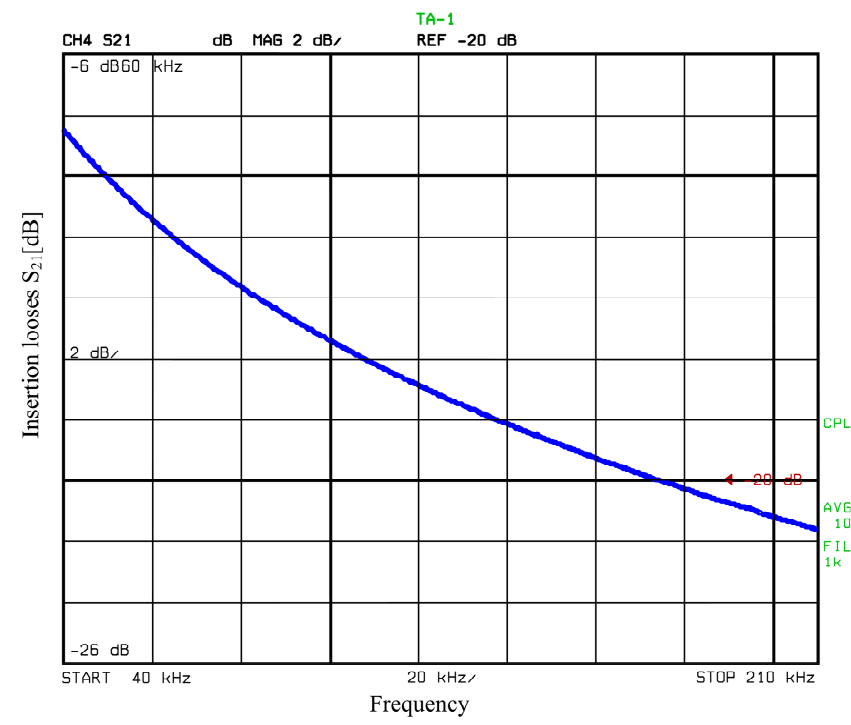

(a)

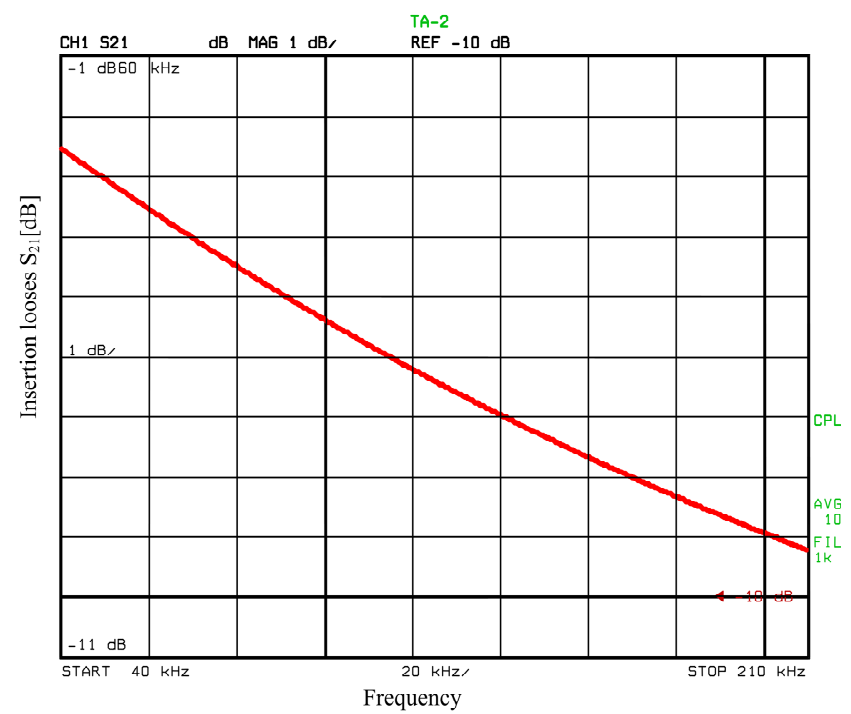

(b)

Fig. 4. Insertion losses versus frequency measured for: a) the type- 1 transformer, b) the type- 2 transformer. The horizontal axis is divided into constant intervals of $20 \mathrm{kHz}$ per division in the interval [40,210] kHz. In subplot (a) the vertical axis is shown with $2 \mathrm{~dB}$ per division from $-26 \mathrm{~dB}$ to $-6 \mathrm{~dB}$, whereas in subplot (b) it is shown $1 \mathrm{~dB}$ per division from $-11 \mathrm{~dB}$ to $-1 \mathrm{~dB}$.

Concerning the imaginary part of the admittance, the type-1 transformer shows an inductive behavior at low frequencies, which becomes capacitive for the highest frequency, i.e. 200 kHz. The type-2 transformer behaves more inductive and the imaginary part of its open-circuit admittance is never positive in the frequency range of interest.

The measured parallel capacitor shows smaller values in the type-2 transformer for frequencies up to $120 \mathrm{kHz}$, exhibiting similar values for large frequencies.

In conclusion, the model parameters estimated for both types confirm that the type-2 transformer outperforms the type-1 one, since the latter presents greater flux leakage, resistive losses, and parasitic capacitance than the former. It is confirmed in the measured insertion losses shown in Fig. 4 a and Fig. $4 \mathrm{~b}$. The type- 1 transformer exhibits an insertion loss in the interval $[7,21] \mathrm{dB}$, whereas it is in the interval $[3,9] \mathrm{dB}$ for the type-2 transformer in the same frequency interval.

\section{3) Proposed model}

The proposed electrical model for the isolation transformer is shown in Fig. 5. This model is generally used for RF transformers [10].

In Fig. 5, the dependent source models an ideal transformer behavior. The model has two inductors: Ls is a series inductor, and $\mathrm{Lm}$ is the magnetizing inductor. The series inductor models the flux leakage, while $\mathrm{Lm}$ is responsible for inducting current in the secondary port.

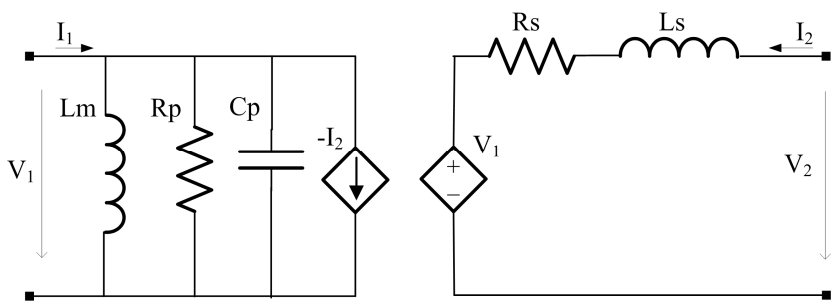

Fig. 5. Proposed model for the isolation transformer
The isolation transformer also exhibits a resistive behavior, which is modeled with two resistors: Rp and Rs. The shunt resistor Rs models the resistive losses in the transformer core. Rs is a series resistor that models the winding losses. Finally, the input parasitic capacitance is modeled by the capacitor $\mathrm{Cp}$.

Table III and Table IV show the adjusted values for the type-1 and type-2 transformers, respectively. A parallelinductive dominant behavior due to $\mathrm{Lm}$ can be observed in both cases. Note that the type-1 isolation transformer presents a higher Lm than the type-2.

Regarding the serial inductor Ls, the type-1 transformer presents values four to six times higher than the type-2, indicating that the flux leakage measured at a given frequency in the range of interest is much higher in the type-1 transformer.

Both resistors, $R s$ and $R p$, increase with the frequency. It can be observed that the losses in the transformer core (modeled as $R p$ ) exhibit a frequency dependency similar to the skin effect.

\section{B. Beacon lamp}

We have analyzed a type L2501-45W-6.6A-EXM halogen lamp (manufactured by Sylvania). The vector network analyzer shows that the lamp basically exhibits a resistive behavior approximately equal to $100 \mathrm{~m} \Omega$ in the whole frequency range under study. Note that this value is ten times smaller than the impedance measured for $50 \mathrm{~Hz}$.

\section{Primary circuit cable}

\section{1) Cable description}

The primary series circuit cable is a screened single-core cable that provides an isolation voltage of $5 \mathrm{kV}$. The copper conductor cross section can vary from $6 \mathrm{~mm}^{2}$ to $8 \mathrm{~mm}^{2}$-AWG. A grounded metal screen surrounds the conductor insulation. This screen protects the cable against electrical discharge phenomena, and gives the cable a higher mechanical strength, 
especially against rodents.

We have selected a FAA L-824 type B compliant cable, and we have performed some measures in the laboratory using a vector network analyzer.

\section{2) Proposed model and results}

Since the shorter wavelength used in PLC is approximately $1.5 \mathrm{~km}$ (equivalent to $200 \mathrm{kHz}$ ) and the cable length is typically greater than the $1 \mathrm{~km}$, a distributed model is proposed, i.e., a lossy transmission line. We assume that the main propagation mechanism in power-line communication is due to a traverse electric magnetic (TEM) propagation mode.

A vector network analyzer has been connected between the core and the metallic shield in order to measure the primary line constants of the lossy transmission line: the distributed resistance $\left(R^{\prime}\right)$, the distributed inductance $\left(L^{\prime}\right)$, the distributed capacitance $\left(C^{\prime}\right)$ and the distributed conductance $\left(G^{\prime}\right)$. These results, measured at $80,120,160$ and $200 \mathrm{kHz}$, are presented in Table V. From these results, we derived the estimated electrical parameters (see Table VI) for the selected range of frequencies. In Table VI $Z_{0}$ is the characteristic impedance, $\alpha$ is the attenuation constant (in $\mathrm{m}^{-1}$ ), $\beta$ is the phase constant, $v_{p}$ is the propagation velocity, and $\alpha$ ' is the attenuation constant (in $\mathrm{dB}$ per $\mathrm{km}$ ).

\section{MODEL VALIDATION}

Once the elements of the power distribution network have been analyzed and modeled, in this section these models are validated. To this end, we have modeled and simulated an airfield ground lighting network using SPICE. The simulation results have been compared to experimental results. The field experiments have been performed in the airport of Seville.

\section{A. Field experiment description}

We have chosen the taxiway edge circuit to carry out the experiments. This circuit is close to the airport terminal, and it is used to guide the pilot to drive the aircraft between the berthing and the parking areas.

The selected circuit has 72 beacons (one beacon working as a transmitter connected to a type- 1 transformer, one beacon as a receiver also connected to a type-1 transformer, and 70 passive beacons connected to the primary circuit through transformers of unknown types). The primary cable is 4500 meters long. The diagram of Fig. 6 describes the experiment, and details the transmitter and receiver beacons.

A signal function generator is used as a transmitter with an output impedance $R g=50 \Omega$, whereas the resistor $R t$ models the receiver side. Transmitter and receiver are connected to the beacons using the following elements:

- a toroidal signal transformer $\left(\mathrm{ST}_{\mathrm{Tx}}\right.$ and $\left.\mathrm{ST}_{\mathrm{Rx}}\right)$ made of ferrite, and designed to provide low distortion and reduced attenuation in the selected frequency band.

- a capacitor $(\mathrm{Cb})$ that works as a high pass filter. It is in charge of presenting high impedance at the power signal frequency $(50 \mathrm{~Hz}$ or $60 \mathrm{~Hz})$, whereas the high frequency currents can flow to the signal transformer.

- an inductor ( $\mathrm{Lb})$ that behaves as a low pass filter (open- circuit in the PLC frequency band), responsible for decoupling the lamp at high frequencies.

The generator $V g$ provides a sinusoidal signal with fixed amplitude $(5 \mathrm{Vpp})$ and tunable frequency. Both, the transmitted $(V T x)$ and the received (VRx) voltages are measured with oscilloscopes connected to $R g$ and $R t$, respectively.

The transmitted beacon is located in the regulator room (Fig. 7) whereas the receiver beacon is ad-hoc installed in one of the IT enclosures in the neighborhood of the regulator room.

TABLE III

MODEL OF THE TYPE-1 ISOLATION TRANSFORMER IN THE FREQUENCY BAND OF INTEREST

\begin{tabular}{|l|c|c|c|c|}
\hline $\boldsymbol{f}[\mathbf{k H z}]=$ & $\mathbf{8 0}$ & $\mathbf{1 2 0}$ & $\mathbf{1 6 0}$ & $\mathbf{2 0 0}$ \\
\hline \hline $\boldsymbol{L m}[\mathbf{m H}]=$ & 5.07 & 4.64 & 4.36 & 3.65 \\
\hline $\boldsymbol{L} \boldsymbol{s}[\boldsymbol{\mu H}]=$ & 812 & 835 & 892 & 1000 \\
\hline $\boldsymbol{C} \boldsymbol{p}[\mathbf{p F}]=$ & 182 & 205 & 213 & 249 \\
\hline $\boldsymbol{R} \boldsymbol{s}=\alpha f^{\beta}$ & \multicolumn{5}{|c|}{$\alpha=1.1 \mathrm{e}-5 \beta=1.38$} \\
\hline $\boldsymbol{R} \boldsymbol{p}=\alpha^{\prime} f^{\beta^{\prime}}$ & \multicolumn{5}{c|}{$\alpha^{\prime}=3.08 \beta^{\prime}=0.58$} \\
\hline
\end{tabular}

TABLE IV

MODEL OF THE TYPE-2 ISOLATION TRANSFORMER IN THE FREQUENCY BAND OF INTEREST

\begin{tabular}{|l|l|l|l|l|}
\hline $\boldsymbol{f}[\mathbf{k H z}]=$ & $\mathbf{8 0}$ & $\mathbf{1 2 0}$ & $\mathbf{1 6 0}$ & $\mathbf{2 0 0}$ \\
\hline \hline $\boldsymbol{L} \boldsymbol{m}[\mathbf{m H}]=$ & 2.43 & 2.05 & 1.80 & 1.67 \\
\hline $\boldsymbol{L} \boldsymbol{s}[\boldsymbol{\mu H}]=$ & 187.8 & 179.3 & 175.4 & 174.4 \\
\hline $\boldsymbol{C} \boldsymbol{p}[\mathbf{p F}]=$ & 71.8 & 138.4 & 215.0 & 249.7 \\
\hline $\boldsymbol{R} \boldsymbol{s}=\alpha f^{\beta}$ & \multicolumn{5}{|c|}{$\alpha=3.22 \mathrm{e}-3 \beta=0.79$} \\
\hline $\boldsymbol{R} \boldsymbol{p}=\alpha^{\prime} f^{\beta^{\prime}}$ & \multicolumn{5}{c|}{$\alpha^{\prime}=1.55 \beta^{\prime}=0.54$} \\
\hline
\end{tabular}

TABLE V

MEASURED TRANSMISSION LINE PARAMETERS FOR THE PRIMARY CABLE

\begin{tabular}{|l|c|c|c|c|}
\hline $\mathbf{f}[\mathbf{k H z}]$ & $\mathbf{8 0}$ & $\mathbf{1 2 0}$ & $\mathbf{1 6 0}$ & $\mathbf{2 0 0}$ \\
\hline $\boldsymbol{R}^{\prime}[\mathbf{\Omega} / \mathbf{m}]$ & $2.12 \mathrm{e}-2$ & $2.47 \mathrm{e}-2$ & $2.66 \mathrm{e}-2$ & $2.94 \mathrm{e}-2$ \\
\hline $\boldsymbol{L}^{\prime}[\mathbf{u H} / \mathbf{m}]$ & $3.60 \mathrm{e}-1$ & $3.47 \mathrm{e}-1$ & $3.45 \mathrm{e}-1$ & $3.44 \mathrm{e}-1$ \\
\hline $\boldsymbol{C}^{\prime}[\mathbf{p F} / \mathbf{m}]=$ & $1.22 \mathrm{e}-1$ & $1.23 \mathrm{e}-1$ & $1.23 \mathrm{e}-1$ & $1.25 \mathrm{e}-1$ \\
\hline $\boldsymbol{G}^{\prime}[\mathbf{S} / \mathbf{m}]=$ & \multicolumn{5}{|c}{$\approx 0$} \\
\hline
\end{tabular}

TABLE VI

ESTIMATED ELECTRICAL PARAMETERS FOR THE PRIMARY CABLE

\begin{tabular}{|l|c|c|c|c|}
\hline $\mathbf{f}[\mathbf{k H z}]$ & $\mathbf{6 0}$ & $\mathbf{1 0 0}$ & $\mathbf{1 4 0}$ & $\mathbf{2 0 0}$ \\
\hline \hline $\boldsymbol{\alpha}\left[\mathbf{m}^{-1}\right]$ & $1.9 \mathrm{e}-4$ & $2.3 \mathrm{e}-4$ & $2.5 \mathrm{e}-4$ & $2.8 \mathrm{e}-4$ \\
\hline $\boldsymbol{\beta}\left[\mathbf{m}^{-1}\right]$ & $2.5 \mathrm{e}-3$ & $4.1 \mathrm{e}-3$ & $5.7 \mathrm{e}-3$ & $8.2 \mathrm{e}-3$ \\
\hline $\mathbf{V} \mathbf{p}(\mathbf{w})[\mathbf{m} / \mathbf{s}]$ & $1.5 \mathrm{e} 8$ & $1.5 \mathrm{e} 8$ & $1.5 \mathrm{e} 8$ & $1.5 \mathrm{e} 8$ \\
\hline $\mathbf{Z}_{\mathbf{0}}[\mathbf{\Omega}]$ & 54.2 & 53.2 & 52.8 & 52.5 \\
\hline $\boldsymbol{\alpha}^{\prime}[\mathbf{d B} / \mathbf{k m}]$ & 1.7 & 2.0 & 2.2 & 2.4 \\
\hline
\end{tabular}




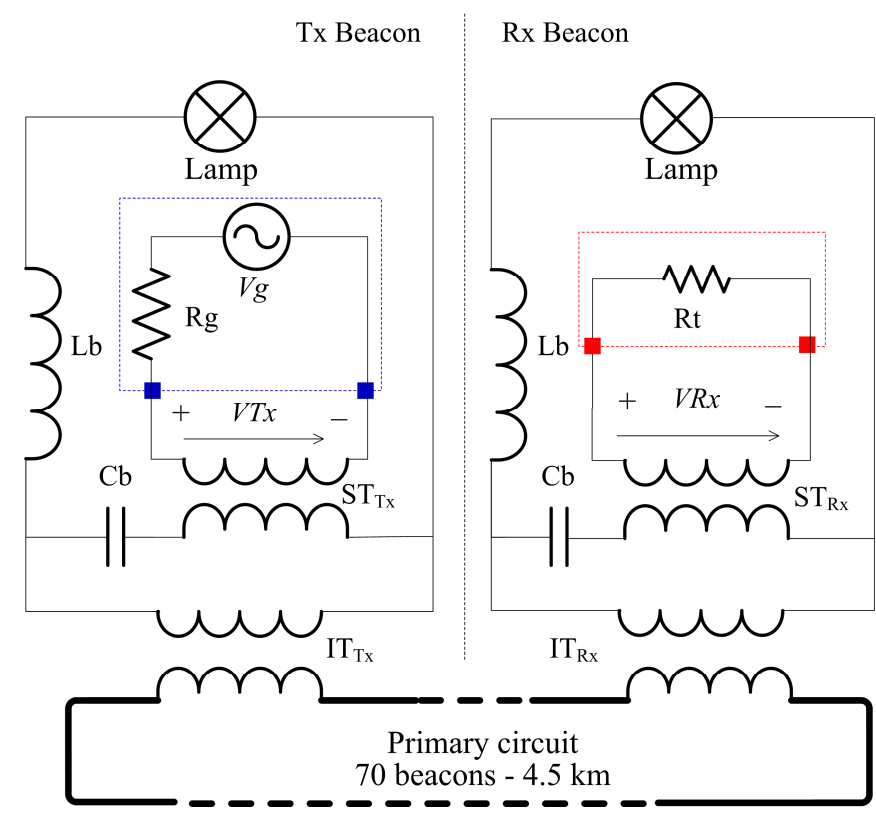

Fig. 6. Field experiments: transmitter and receiver detailed schematics.

All the experiments have been done with the current regulators turned-off and in short-circuit. Furthermore, the other 70 beacons are composed of one lamp directly connected to the isolation transformer. Fig. 7 shows a photo of the test benchmark.

\section{B. Simplified model}

The scheme presented in Fig. 6 has been simulated in SPICE using the models proposed in Section III. The 70 passive beacons (without PLC units) are assumed to be equally distributed along the primary circuit. Hence, the primary cable is equally chopped in 60 meters long segments. Every segment is modeled as a lossy transmission line.

In the 70 passive beacons the lamp is directly connected to the isolation transformer. Note that this situation is only useful to emulate this experiment. In the final application, every beacon has the same scheme depicted in Fig. 6.

\section{Results}

Fig. 8 shows the transmitter input voltage $(V T x)$, the received output voltage $(V R x)$, and the channel attenuation $(V T x / V R x$ in $\mathrm{dB})$ versus frequency. In this figure the values provided by SPICE simulations are compared to the experimental results measured in the airport. It can be concluded that the proposed model is especially valid for the center frequency, i.e. around $140 \mathrm{kHz}$. In the lower and the upper end of the frequency interval some small differences can be observed.

Another important result is that the attenuation is mainly due to the isolation transformers. For example, at $120 \mathrm{kHz}$, the measured attenuation is $44 \mathrm{~dB}$ : the isolation transformers attenuate the PLC signal $38 \mathrm{~dB}(19 \mathrm{~dB}$ in the $\mathrm{Tx}+19 \mathrm{~dB}$ in the $\mathrm{Rx}$ ), the transmission line $2 \mathrm{~dB}$ (which means that they are about $1 \mathrm{~km}$ apart), and the signal transformers and filters $4 \mathrm{~dB}$ (from the measurements taken in the laboratory).

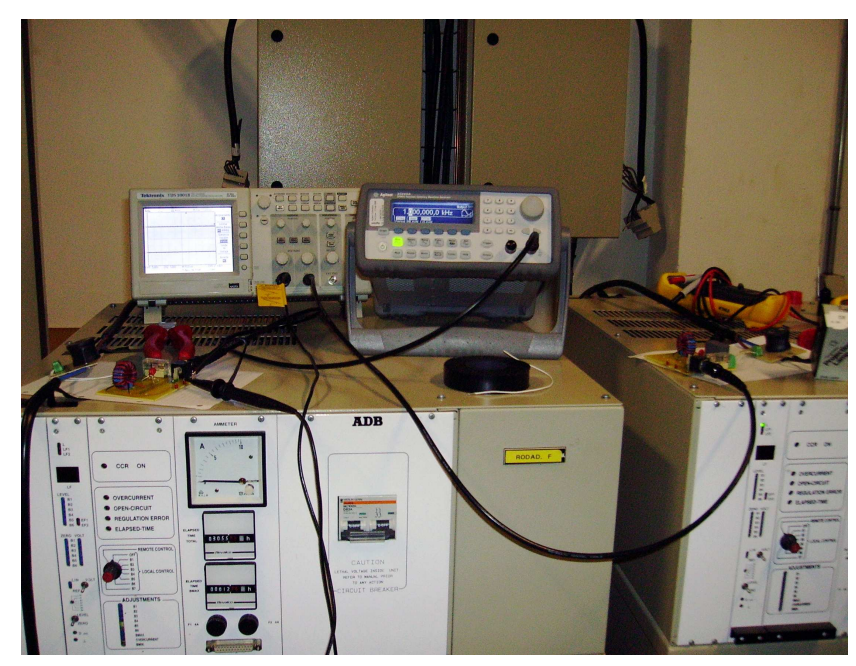

Fig. 7. Field experiments: transmitter located in the regulator room onto the constant current regulator.

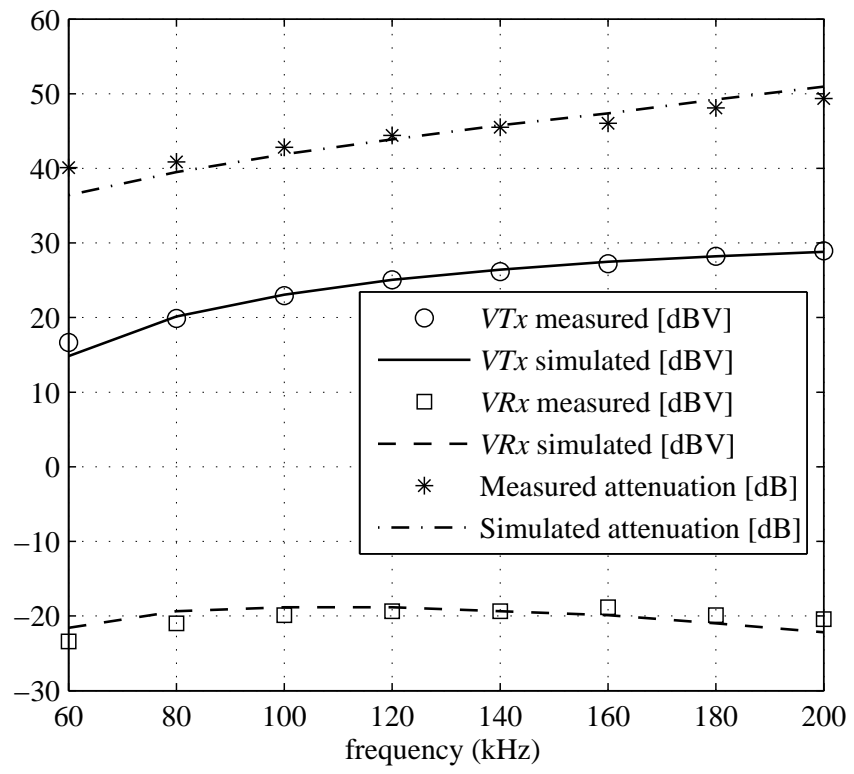

Fig. 8. Simulation versus experimental results.

\section{THE PLC LINK}

This section proposes a simple mechanism for power budget calculation in PLC links over airfield ground lighting systems. The proposed method is suitable for narrow-band PLC modems working in the $(60-200) \mathrm{kHz}$ frequency band. In addition, a set of rules is also given in order to help designers to develop and optimize these applications.

\section{A. Power budget calculation}

The elements which introduce attenuation in the link are: the isolation transformers, cables, coupling circuitry (i.e., those circuits in charge of injecting or recovering the PLC signal) and other circuitry (lamps, CCR and protections). 
The largest attenuation is due to the isolation transformers. This attenuation exhibits a strong dependency on the carrier frequency (which is a design parameter and can be modified according to the system requirements). Moreover, the attenuation depends on the transformer type (which is unlikely to be replaced). The transformer attenuation can vary from $6 \mathrm{~dB}$ (at the lowest frequency using the analyzed type-2 transformers in both extremes of the PLC link) to $42 \mathrm{~dB}$ (with the carrier frequency close to $200 \mathrm{kHz}$ using the type-1 transformer in both, the transmitter and the receiver).

Regarding the cable attenuation, the distance between beacons rarely exceeds $100 \mathrm{~m}$ including both, the primary and the secondary segments. Therefore, if a beacon-to-beacon communication is considered, the expected attenuation is insignificant (in the worst case it is smaller than $0.25 \mathrm{~dB}$ ). Otherwise, if the communication is established between the CCR room and the farthest beacon of the circuit, the total attenuation can be comparable to the isolation transformer attenuation.

Filters and, specially, coupling circuitry are responsible for a power attenuation that could be large, and therefore, they should be carefully considered in the design.

\section{B. Design considerations}

In large AGL circuits the total attenuation between the CCR room and the farthest beacon can be as large as $80 \mathrm{~dB}$. The PLC modems have to be able to manage theses large attenuation by means of PLC repeaters.

A frequency selective behavior is expected, as the impedance discontinuities along the circuit produce signal echoes with different amplitude and phase, producing constructive or destructive interference at the receiver input. As a result, shadow areas are likely to appear in locations other than the circuit end points. Reducing the carrier frequency, installing repeaters, selecting a robust modulation scheme like OFDM (orthogonal frequency division multiplexing) and making sure that the primary grounded shield is not chopped, should help to solve this problem.

\section{Noise}

Different kinds of noise sources present in the AGL powerline have not been considered either in this paper or in the bibliography. Some measurements carried out during the field experiments showed that the main noise source is the constant current regulator.

The CCR generates a high amplitude synchronous impulsive noise which degrades the signal to noise ratio during a fraction of the power signal period $(20 \mathrm{~ms}$ in $50 \mathrm{~Hz})$. To reduce its impact on the communication performance, the PLC bursts should avoid those intervals where the impulsive noise is dominant.

\section{CONCLUSIONS}

Reusing the AGL power distribution circuit to transmit control and supervision data is a non-conventional PLC application that the authors survey in this paper. Due to the low bandwidth requirements of this application, narrowband PLC modems in the range 60 to $200 \mathrm{kHz}$ are considered to be suitable for this application.

The main elements of an AGL circuit have been analyzed and measured in laboratory to obtain their SPICE models. Using these models a complete PLC link model has been obtained which has been validated by means of field experiments.

In our analysis, we have realized that the attenuation is mainly due to the isolation transformers, which highly depends on the type of transformer used in the airport. In addition, the cable attenuation could be dominant for transmission between the CCR room and the farthest beacons.

Finally, a set of design considerations are given to help designers to optimize PLC networks for this application.

\section{REFERENCES}

[1] Zhang Jundong, Ren Guang and Sun Peiting, "A monitoring and control system based on local network for airfield lighting," in Proc. 2001 International Conferences on Info-Tech and Info-Net, vol. 4, pp. 192195, Oct. 2001

[2] K. Lee, "Integration of aviation lighting system and computer controlled monitoring system," in Proc. IEEE International Conference on Systems, Man, and Cybernetics 1996, vol. 2, pp. 1132-1137, Oct. 1995.

[3] International Civil Aviation Organization ICAO. Aerodrome Design Manual, part 4, visual aids. $4^{\text {th }}$ edition, 2004.

[4] Federal Aviation Administration FAA, Airport Safety and Standars (ASS). Design and installation details for airport visual aids (150/534030D), FAA, Sep. 2008.

[5] Airfield Smart Power (ASP) from Safegate group.

[6] E. Liu, Y. Gao, G. Samdani, G. Mukhtar and O. Korhonen, "Powerline communication over special systems," in Proc. 2005 IEEE International Symposium on Power-line Communications and Its Applications (ISPLC'05), pp. 167-171, April 2005.

[7] G. Dickmann and N.J. Fliege, "Digital signal processing for multicarrier data transmission on phase-controlled power-lines with nonlinearities," IEEE International Symposium on, Circuits and Systems (ISCAS 95), vol. 2, pp. 889-892, Apr. 1995.

[8] International Civil Aviation Organization ICAO. Aerodrome Design Manual, part 5, electrical systems, $1^{\text {st }}$ edition, 1983.

[9] Federal Aviation Administration FAA, Airport Safety and Standars (ASS). Specification for series to series isolation transformers for airport lighting system (150/5345-47B), 2005.

[10] C. Trask, "Wideband transformers: an intuitive approach to models, characterization and design," Applied Microwave \& Wireless, pp. 3041, Nov. 2001.

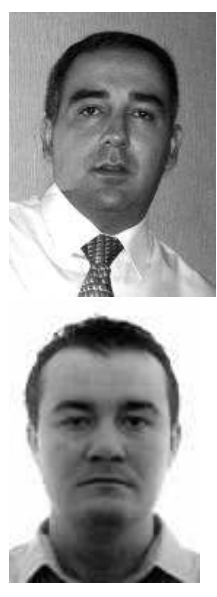

\section{BIOGRAPHIES}

Joaquín Granado was born in Córdoba (Spain) in 1973 and received his Master and Ph.D. degrees in Telecommunication Engineering in 1999 and 2005, both from the University of Seville.

$\mathrm{He}$ is currently with the Electronic Engineering Department of this University. His research interest is in wireless and wired communication techniques, especially in OFDM systems

Jorge Chávez was born in Seville, Spain. He received his Master and $\mathrm{Ph}$. D. degrees in Electrical Engineering from the University of Seville, (Spain) in 1992 and 1996 respectively.

Since 1992 he has been with the Department of Electronic Engineering, Escuela Superior de Ingenieros, University of Seville. His current research interests include analog and digital signal processing, communication systems, and broadband communications systems design including PLC, DVB$\mathrm{T}$ and DVB-H. 


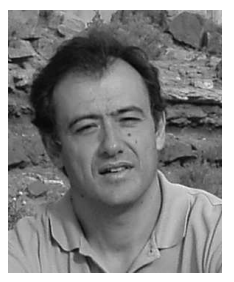

Antonio Torralba was born in Seville (Spain). He received a Master (1983) and Ph.D. (1985) degrees in Electrical Engineering, both from the University of Seville. In 1983 Dr. Torralba joined the Electronic Engineering Dept., University of Seville, where he presently maintains a Full Professor position.

Prof. Torralba is author of 60 papers in international journals and has participated in, and in many cases led, more than 40 research projects with National and European funding.

His research activity is mainly focused in the design of circuits and systems for wireless and wired communication.

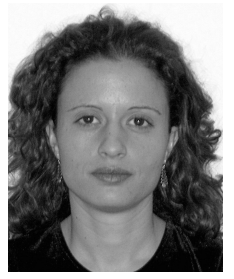

Ana Cinta Oria Oria was born in Huelva, Spain. She received his Master and Ph.D. degrees in Telecommunication Engineering in 2005 and 2010, both from the University of Seville. Since 2005, she has been with the Department of Electronic Engineering, Escuela Superior de Ingenieros, University of Seville. Her current research interest is in OFDM systems, especially in digital video broadcasting systems. 